\title{
Tools and technologies needed for conducting planetary field geology while on EVA: Insights from the 2010 Desert RATS geologist crewmembers
}

\author{
Kelsey Young a,*, José M. Hurtado Jr. ${ }^{\mathrm{b}}$, Jacob E. Bleacher ${ }^{\mathrm{c}}$, W. Brent Garry ${ }^{\mathrm{d}}$, Scott Bleisath ${ }^{\mathrm{e}}$, \\ Jesse Buffington ${ }^{\mathrm{f}}$, James W. Rice Jr. ${ }^{\mathrm{c}}$ \\ a Arizona State University, School of Earth and Space Exploration, Bateman Physical Sciences Center F-wing, Room 686, Tempe, AZ 85287-1404, United States \\ ${ }^{\mathrm{b}}$ University of Texas at El Paso, Department of Geological Sciences, 500 West University Avenue, El Paso, TX 79968-05555, United States \\ ${ }^{c}$ Planetary Geodynamics Laboratory, Code 698, NASA Goddard Space Flight Center, 8800 Greenbelt Road, Greenbelt, MD 20771, United States \\ ' Planetary Science Institute, 1700 East Fort Lowell, Suite 106, Tucson, AZ 85719, United States \\ e NASA Glenn Research Center, 21000 Brookpark Road, Cleveland, OH 44135, United States \\ ${ }^{f}$ EVA Hardware Development Tools and Equipment Branch (EC7), NASA Johnson Space Center, 2101 NASA Parkway, Houston, TX 77058, United States
}

\section{A R T I C L E I N F O}

\section{Article history:}

Received 1 July 2011

Received in revised form

6 October 2011

Accepted 24 October 2011

\section{Keywords:}

Field geology

Sample collection

Planetary analog

EVA technology

EVA tools

Planetary field geology

\begin{abstract}
A B S T R A C T
The tools used by crews while on extravehicular activity during future missions to other bodies in the Solar System will be a combination of traditional geologic field tools (e.g. hammers, rakes, sample bags) and state-of-the-art technologies (e.g. high definition cameras, digital situational awareness devices, and new geologic tools). In the 2010 Desert Research and Technology Studies (RATS) field test, four crews, each consisting of an astronaut/engineer and field geologist, tested and evaluated various technologies during two weeks of simulated spacewalks in the San Francisco volcanic field, Arizona. These tools consisted of both Apollo-style field geology tools and modern technological equipment not used during the six Apollo lunar landings. The underlying exploration driver for this field test was to establish the protocols and technology needed for an eventual manned mission to an asteroid, the Moon, or Mars. The authors of this paper represent Desert RATS geologist crewmembers as well as two engineers who worked on technology development. Here we present an evaluation and assessment of these tools and technologies based on our first-hand experience of using them during the analog field test. We intend this to serve as a basis for continued development of technologies and protocols used for conducting planetary field geology as the Solar System exploration community moves forward into the next generation of planetary surface exploration.
\end{abstract}

(c) 2011 Elsevier Ltd. All rights reserved.

\footnotetext{
Abbreviations: CFN, Crew Field Note; COTS, commercial off-the-shelf; DSB, Documented Sample Bag; DVR, digital video recorder; EVA, Extravehicular Activity; EVAIS, Extravehicular Activity Information System; GIS, Geographic Information System; GPS, global positioning system; $\mathrm{HCl}$, hydrochloric acid; HD, high definition; IVA, Intravehicular Activity; LEM, lunar excursion module; LiDAR, Light Detection and Ranging; LRV, Lunar Roving Vehicle; NASA, National Aeronautics and Space Administration; RATS, Research and Technology Studies; RFID, radio-frequency identification; SEV, Space Exploration Vehicle; XRF, X-ray fluorescence

${ }^{*}$ Corresponding author. Tel.: +1 7035776562 .

E-mail address: Kelsey.E.Young@asu.edu (K. Young).
}

\section{Introduction-overview of the Desert RATS 2010 operation}

The 2010 Desert Research and Technology Studies (RATS) field test consisted of two seven-day traverses of two small, pressurized rover prototypes. All fourteen days of the mission were conducted with both vehicles operating simultaneously, moving through a pre-determined traverse plan, with a crew 
change on day seven. A crew of two, one commander/ engineer and one geologist with extensive field experience, operated each SEV (Space Exploration Vehicle). Three out of four commanders were experienced National Aeronautics and Space Administration (NASA) astronauts with at least one Space Shuttle flight. The geologists were selected from various NASA centers and academic institutions based on their familiarity with working in the field. Each crew therefore had the range of skills necessary to deal with both mission operations and science activities. Another benefit of the 2010 crew structure and mission duration was that a total of eight crewmembers (four each week) were able to test the technology and protocols, providing more input to the engineering teams. For a complete description of both the 2010 Desert RATS field test and preceding tests, see Kosmo et al. (this issue) [1].

Each traverse day was marked by several (1-4) extravehicular activities (EVAs) of varying duration, where each crew egressed from their rover for "boots-on-the-ground" scientific data collection. The crews collected samples and recorded their observations using a suite of equipment mounted on both the aft deck of the SEV (Fig. 1) and the mock-up spacesuits (Fig. 2; for details on protocols and procedures while on EVA, see Hurtado et al. (this issue) [2]). These ranged from the simple hand tools used in sample collection to prototype imaging and computing technologies designed to document crew observations while on EVA. This paper seeks to describe these tools and technologies and put forth our experiences with their utility. The authors of this paper represent Desert RATS geologist crewmembers as well as two engineers who developed the technologies described here. We focus on the technology used in the 2010 Desert RATS test, present lessons learned from the 2010 simulated mission, and offer suggestions for future tests and real mission scenarios.

\section{History of planetary field geology}

The only example of humans conducting field geology on another planetary surface is the Apollo program

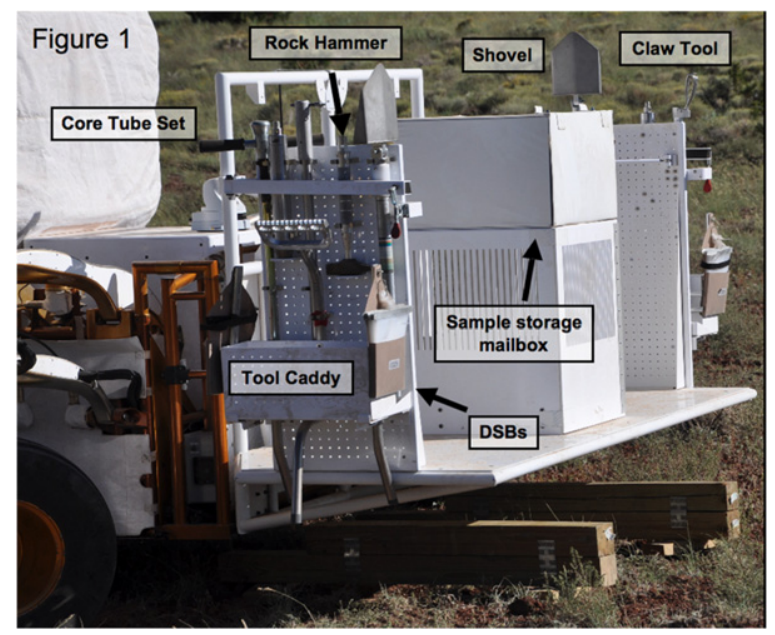

Fig. 1. The aft deck of the SEVs held a variety of tools used by the crew on EVA. Tool functions are described in Section 3.1.

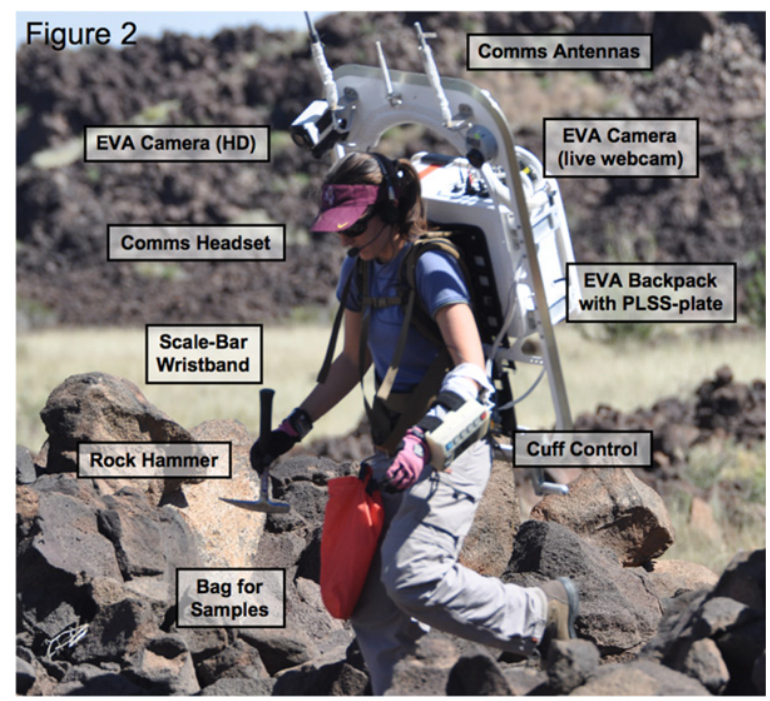

Fig. 2. A 2010 Desert RATS geologist crewmember traverses over a lava flow while on an EVA. Some of the tools available for EVA are shown here. The PLSS (portable life support system) plate is the large rectangular metal outline that allows the pack to fit into the suitports on the SEV, allowing for a safe and relatively quick ingress into the pressurized rover.

(1969-1972). During these six surface missions, twelve astronauts conducted a series of EVAs on the Moon, collecting samples and making scientific observations that have revolutionized our understanding of lunar geology and left us with a wealth of lessons learned about developing technology for planetary field geology. This is, however, our only data point in conducting manned science operations on another planetary surface. Combining these lessons learned during Apollo into equipment that reflects the technological advancements that have occurred since the early 1970s will give us the best chance of increasing the efficiency of scientific data collection on other planetary bodies.

\subsection{Apollo era field geology}

Due to the short time each crew was able to spend on the lunar surface during the six Apollo missions (ranging from $2 \mathrm{~h}, 14$ min during Apollo 11 to roughly $22 \mathrm{~h}$ during Apollo 17) [3], EVA activities were tightly scheduled. Each Apollo mission had very specific mission objectives, and EVA tasks were limited to only those addressing the predetermined objectives. Science teams worked in advance to develop traverse plans for each landing site, and the astronauts were instructed to follow these plans extremely carefully. As a result, there was little flexibility for modifying or improvising plans while on EVA [4].

In order to assist the astronauts in collecting samples, installing experiments, and operating on the lunar surface, engineering teams designed a suite of tools for the Apollo missions [5]. EVA sample collection tools included tongs, a scoop, a rake, and a hammer, all made to assist the astronaut in isolating a sample for bagging and returning it to Earth. Core tubes and an electric drill (only deployed on Apollo 15-17, also known as the J-missions), were used to sample material from beneath the surface. Samples were stored in individual bags and containers 
marked with unique numbers that helped the astronauts and scientists on Earth connect a sample to its collection site. Once they had documented, photographed, collected, and bagged a sample, the astronauts stored it in a larger bag clipped to the suit of their partner because it was too awkward to reach behind to access their own suit's storage bag. This resulted in the two astronauts having to remain quite close to one another while on EVA, instead of potentially separating to cover more ground (Apollo flight rules also mandated that the crewmembers remain relatively close to one another) [3]. Finally, the collected samples were stowed in larger boxes on board the lunar excursion module (LEM) for transport back to Earth [5].

The Lunar Roving Vehicle (LRV) was introduced for the Apollo J-missions. As a small, unpressurized rover, the LRV provided a way to drastically increase the distance the astronauts were able to travel away from the LEM, therefore increasing the amount of observational data each crew was able to obtain. For example, Apollo 14 covered a total of $\sim 3.5 \mathrm{~km}$, compared to the $\sim 28 \mathrm{~km}$ traversed by Apollo 15 and the $\sim 35 \mathrm{~km}$ traveled by the Apollo 17 crew [3]. A predecessor to the Desert RATS SEV, the LRV provided transport to the astronauts and also supplied sample and tool stowage while on a traverse. The LRV also served as a platform for a color video camera used to document activities at each EVA stop, which served as the precursor to the cameras that were used in the 2010 Desert RATS field test. The distance covered in the J-missions, as well as the added stowage and data collection ability, show clearly that the LRV enabled the crews to accomplish more with added efficiency [6]. However, the LRV did not provide the crew with a respite from their pressurized suit, which is a major advantage of the SEV [7].

\subsection{Flexibility during the Apollo surface missions}

The combination of limited time on the surface and stringent safety constraints led to strict traverse plans during the Apollo era. Although highly effective in covering the designated landing sites and meeting mission objectives, the overall exploration strategy left room for future development. An innovation inspired by scientific exploration on Earth and which is being embraced by field tests like Desert RATS is the concept of "flexecution", wherein the crew is given much more flexibility and autonomy in how plans are executed to meet mission objectives [8-10]. While initial traverses were designed using remote sensing data to meet mission science objectives, the Desert RATS crews were given the freedom to make real-time changes to the planned tasks based on their field observations, re-prioritization of science objectives, and unanticipated discoveries. Since future crews, especially those that will visit Mars (where there will be a substantial time delay in communications), will have complete autonomy in the field, this clearly highlights the need for crews to be extensively trained and experienced in conducting field geology. It is crucial that crews have first-hand experience and confidence in making realtime decisions in the field on Earth that can be extrapolated to decisions they will make in a real mission setting. Harrison Schmitt is the only geologist to ever visit another planetary body, and his work during Apollo 17 provided scientists on Earth with vital knowledge of lunar geology $[10,11]$. It should be noted that to be able to flexecute a traverse, crews also need a high-level understanding of the engineering aspects of the mission (for the same reason that such an understanding of geology is needed), and they will require technology that enables the freedom to flexecute rather than be tied to a strict timeline.

\subsection{Links between Desert RATS and terrestrial field geology}

Terrestrial field geologists endeavor to understand the developmental history of an area, including the geologic and geomorphic processes responsible for shaping that history. This story is recorded in the rock record, and a trained field geologist must, therefore, be highly attuned to subtleties of the Earth's surface morphology, the composition of geologic materials (rocks, soils, etc.) on the surface and in the subsurface, and the geometric and structural relationships amongst all these features. As a field geologist works to develop an understanding of an area of geologic interest, they look to all the data available for the site and execute multiple field excursions to both map and collect samples. Terrestrial geologists enjoy the luxury of time, easy access, and relatively easy working conditions when accomplishing their work. Yet, as we move to other planetary surfaces, including those of asteroids, a number of other conditions must be considered in planning surface operations (such as microgravity, communications delays, high costs, limited time on the surface, etc.).

When examining potential tools for use in planetary geologic fieldwork, it is instructive to examine the tools that terrestrial field geologists use on Earth. Basic field geology tools include a rock hammer; bags and containers to store samples; a hand lens for examining small samples under magnification; dilute hydrochloric acid to test for the presence of carbonates; a camera to document outcrops, samples, and geologic context; a scale bar for photographs taken in the field; writing implements to take notes and draw sketches; and a map for collecting geospatial information. The latter is now often in the form of a digital mapping tool equipped with a GPS (global positioning system) receiver. Several of these tools, including the rock hammer, sample bags, camera, and scale bar, are fairly simple to recreate in a planetary surface mission environment, and Desert RATS 2010 had all of these items accessible to the crew while on EVA.

While tools like the hammer and camera are fairly easy to adapt for a planetary environment, certain items used by terrestrial field geologists are not adaptable or even necessary for the missions Desert RATS simulates. For example, the dilute hydrochloric acid $(\mathrm{HCl})$ used by many field geologists may not be the best choice to use in space since it is likely that low atmospheric pressure will change the reaction between the acid and the rock. Acid is also a safety issue while in a pressure suit, and, as a consumable, it is a poor candidate to take on a mission 
where mass will be a precious commodity. In addition to this, diagnostic $\mathrm{HCl}$ is an extremely inflexible tool because, except for identifying carbonates, there is little else that the acid can be used for. Nevertheless the basic purpose of carrying $\mathrm{HCl}$ to use in the field is to help identify rock type and chemistry, so some equivalent is needed here. Handheld, in-situ, analytical geochemical instruments are being developed (such as the handheld $\mathrm{X}$-ray fluorescence (XRF) spectrometer discussed in Evans et al. (this issue) [12]), which could take the place of acid and provide higher-resolution, real-time data about a wider range of rock types and their geochemistry in the field.

The capabilities of some tools, such as the hand lens, will need to be modified for use in a spacesuit. The hand lens is typically held directly next to the eye, with the sample nearly touching the lens on the other side, allowing the terrestrial field geologist to look at a detailed and magnified view of the sample. This close proximity is impossible while wearing a helmet, but the hand lens is a crucial tool that should be part of any planetary surface mission. We hope to incorporate this technology into future tests (see Section 4).

Documenting crew observations and their location on a planetary surface is critical, a lesson learned in the Apollo missions [3]. Technologies that aid in compiling field notes and geologic mapping are absolutely crucial to field geologists. As discussed by Hurtado et al. [2], geologic observations of context and geospatial relationships are as important as the samples collected during EVAs, if not more so. Therefore, geologists must have the ability to constantly document what they are seeing and record their multiple working hypotheses about the geology using, for example, sketch maps and personal notes. Astronauts, hampered with their bulky spacesuits and gloves, are not able to transcribe anything with their hands, so technologies such as voice-activated systems must be employed. Desert RATS is making a concentrated effort in this area with the Crew Field Note (CFN) technology [2], which allows the crewmember to press a button to start and stop the recording of time- and geotagged audio notes linked to images/video (Fig. 5).

Finally, we must consider that field geologists working on Earth have the luxury of their senses (smell, touch, taste, etc.), which are very commonly used in assessing a site. There is no analogous technology for planetary exploration at this time. There is an increasing possibility that handheld, in-situ, real-time analytical instruments such as the XRF can help to fill this gap. Future Desert RATS tests hope to include this technology in the hope of mitigating the problem of lack of traditional senses.

\section{Desert RATS 2010 EVA technology}

During the 2010 field test, each two-person crew completed a series of EVAs designed to collect samples and make observations about the test site. Engineers designed a suite of tools for the crews to assist them in these tasks. A pre-determined and practiced set of EVA protocols was created to ensure that each sample and observation was recorded and the resulting data could be easily accessed, analyzed, and curated by the science team back on "Earth" [2]. Several types of tools were available to the crew during this test. Tools were stored on the aft deck of each SEV (Fig. 1) and on the mock-up spacesuits (Fig. 2). The 2010 geologic toolkit was designed to facilitate the collection of samples and geologic context data while, at the same time, minimizing the burdens placed on the crew in terms of EVA mobility; logistical management of tools and samples; and tracking of individual samples and associated data for curation purposes. To this end, the tools were built and organized as a sample collection system intended to streamline the acquisition, recording, handling, packaging, and return of the traverse's findings.

\subsection{Aft deck tools}

Individual samples could be acquired by several methods: picking up loose clasts by hand, at times with the use of tongs (in anticipation of potential waist/lower torso mobility limits in future spacesuits); subsurface material obtained using core tubes; loose material such as soil excavated with a shovel; and in-situ rock fragments liberated from an outcrop using a hammer. Regardless of the sample acquisition method, once a sample had been captured, it was placed in an individually numbered "Documented Sample Bag" (DSB [13]). During sample acquisition, crewmembers followed explicit protocols on the photography of samples, description of the samples and their context, and recording of the contextual data (for details on these sampling and observational procedures, see Hurtado et al. [2]). Specifically, the various acquisition tools, such as the hammer, were designed with color and length scales so that they could also be used as reference markers in the context photographs (tools shown in Figs. 1 and 2). A variety of other scale bars were placed in strategic places in order to capture size information in the field-ofview of every photograph taken of samples [13]. For example, each crewmember wore a wristband on EVA that showed a scale and color bar (Fig. 2). When the crewmember was imaging a sample, the field-of-view of the backpack cameras (described in Section 3.2) captured this scale bar to show the Science Backroom the size of the sample. Alternatively, if a photograph was taken that showed the sample bag in the field-of-view along with the sample, the size could be determined because of the known size of the sample bag. Hurtado et al. [2] provide a detailed description of these sample collection procedures.

Once documentation was complete, the sample was placed in its bag and then stowed for the remainder of the EVA in a tool caddy (Fig. 3) that was intended to allow the crew to hand carry a single EVA's worth of samples and DSBs across the surface [13]. Once this caddy was full or the EVA was over the crew returned to the aft deck of the rover, where additional curation tasks were carried out [2]. A spring scale used to measure the mass of the samples collected during the EVA facilitated these tasks (Fig. 4). After all samples collected by both crewmembers were weighed and collected on the aft deck, the crew stowed the samples in a sample stowage "mailbox" (Fig. 4) for transport on the SEV for the remainder of the 7-day traverse. Each SEV 

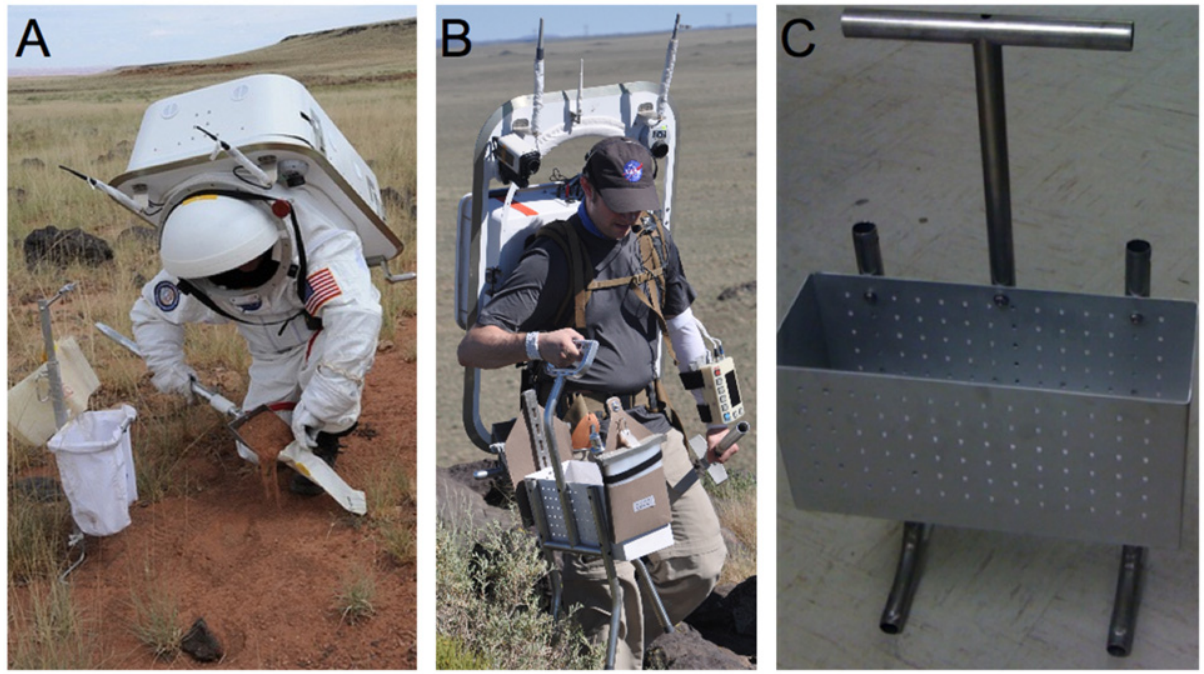

Fig. 3. (A) The tool caddy from the 2009 Desert RATS field test, while light and easy to carry, was not weight bearing. (B, C) The 2010 model is weight bearing, but proved ungainly to manage in the field, often snagging on the ground and hitting the crewmembers' shins and knees. Notice the crewmember holding the caddy up to provide ground clearance during EVA, which is fatiguing to the arm during long walks. We conclude that neither model was ideal. This item must continue to evolve, as it has during Desert RATS tests, and/or new methods of sample and tool stowage should be explored in future field tests.
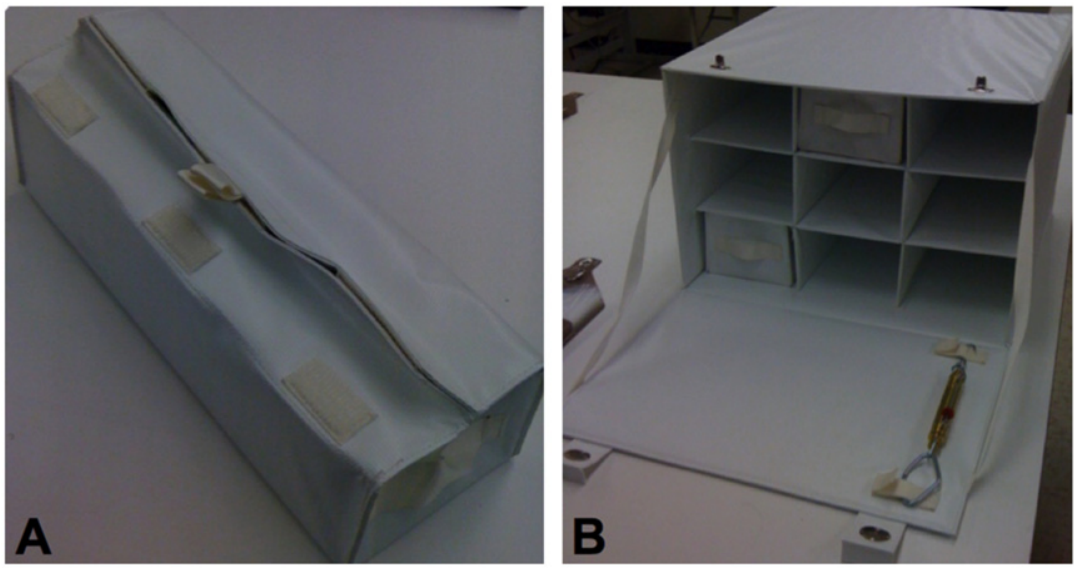

Fig. 4. The sample stowage mailbox. (A) Small, numbered, soft-sided boxes held the DSBs. These boxes would later be placed in the larger sample stowage mailbox depicted in (B). After each EVA, the crew would put all of the collected samples into soft boxes and record a CFN detailing which DSBs went into which box. They would then weigh the filled soft boxes with the spring scale shown in (B). After the weights were recorded in a CFN, the filled soft boxes were placed in the sample stowage mailbox, where they remained until return to Earth or until needed for curation purposes or in-situ analyses [2].

mailbox contained 9 smaller, soft-sided boxes that were each labeled with a unique identifier. Depending on the size of the sample, these smaller boxes could be filled with as many as $8-10$ samples [13]. As the crew loaded each DSB into one of these labeled boxes, they recorded a CFN detailing where each DSB was stored so both the Science Backroom and the crew would know where to look if they needed to retrieve a sample at any point. At the end of the mission, decisions were made, with guidance from the Science Backroom, to preferentially select (or discard) samples based on their individual priority and science return value (i.e. "high-grading" [12]).

\subsection{Backpack technology}

Instead of a high-fidelity, pressurized spacesuit, a lower-fidelity, "shirt-sleeve" backpack was worn while performing EVAs (Fig. 2). The backpack held the following: a pair of cameras, one mounted to each shoulder; a GPS receiver and antenna; and communications equipment, including antennae, batteries, and avionics. Among the avionics inside the backpack was the EVA Information System (EVAIS) developed by the NASA Glenn Research Center. EVAIS is described in more detail in the following section and also includes a small, flat-panel computer 


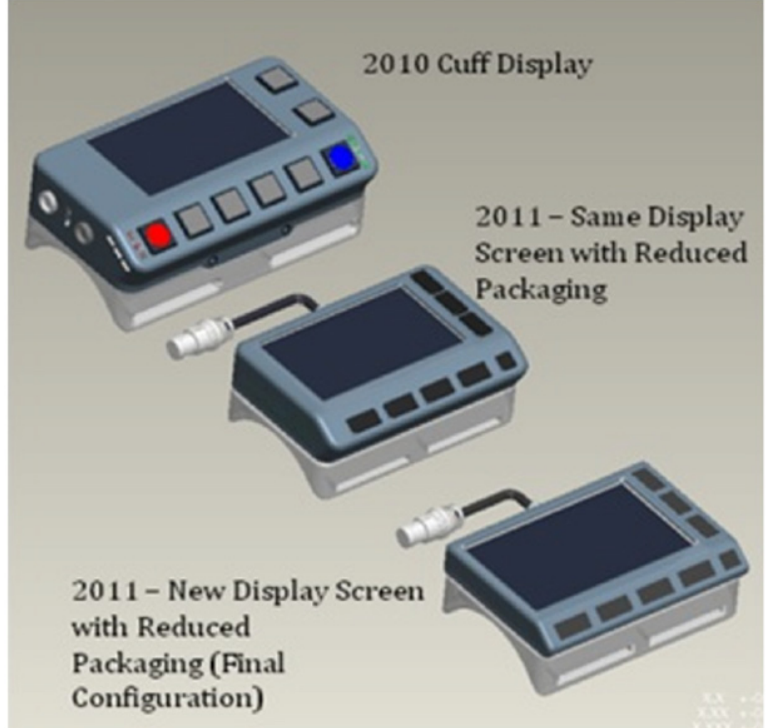

Fig. 5. The cuff display size has been optimized for Desert RATS 2011. Compare the 2011 version with the 2010 cuff display in Fig. 2.

display on the crewmember's wrist (the "cuff display") and a high definition (HD) video camera mounted on the backpack (Fig. 2). While we recognize that traditional GPS technology will not be available in a planetary exploration environment, we use it here as a proxy for an eventual relative positioning technology that will serve to allow astronauts to keep track of sampling locations and traverse paths.

There were two cameras on the 2010 field test suits, one on each shoulder. One was a live web camera mounted on the left shoulder, which was constantly streaming video to the Science Backroom. This web camera was a remnant of the 2009 field test, where it was the only source of real-time video data from a crewmember to the Science Backroom for documenting crew activities on EVAs. The camera on the right shoulder was an HD video camera added for the 2010 field test used for capturing CFNs and was controlled using the EVAIS cuff interface (Fig. 5).

\subsubsection{Cuff control}

The EVAIS cuff display (shown in Figs. 2 and 5) stores and displays procedures, EVA timelines, and traverse maps for the Desert RATS crewmembers. The crew can access procedures as needed to operate their suit systems and to perform EVA tasks. Rapid and easy access to this information is very important for a crewmember on EVA. For any space exploration mission beyond Earth orbit, such as missions to the Moon, near-Earth asteroids, or Mars, communication with flight controllers on Earth may not always be available or may be significantly delayed. The EVAIS enables the EVA crew to manage their activities autonomously and makes flexecution possible.

In addition to displaying procedural information, the EVAIS allows an EVA crewmember to record still photographs and CFNs with the HD video camera. Using a dedicated button on the cuff display, the crewmember activates the camera and built-in digital video recorder (DVR) to record CFNs. The crewmember then gathers a rock sample, holds it up in front of the camera, and provides a verbal description to accompany the video by speaking into a headset microphone [2]. In a similar fashion, the crewmember utilizes the EVAIS to capture high-resolution photographs of rock samples. A commercial off-the-shelf (COTS) GPS contained in the backpack provides a location stamp for all CFNs and still images recorded with EVAIS. The CFNs were downloaded at the end of a traverse day for analysis by the science support team [14].

\subsubsection{Communications equipment}

Each crewmember wore a headset equipped with a microphone while on EVA, which enabled two-way communication with the Science Backroom. Communication between crewmembers was also done through the headset on the occasions where the crew separated while on EVA. Flight rules dictated that the crew could not separate without functioning communications with each other, so this technology played a central role in boots-on-theground operations and the ability to flexecute.

The microphone was also used to record audio data for CFNs. The resulting audio and video database, including both real-time and CFN data, was of crucial importance to the Science Team as it contained the narrative of all scientific observations made by the crew [14]. Furthermore, the shoulder-mounted cameras provided a way to communicate with the Science Backroom. The non-HD camera had a live video-feed to the Science Backroom, which enabled them to see, in real-time, what the crew was seeing. The feedback that the Science Backroom could provide to the crew as a result of the real-time video was integral in monitoring EVA activities and for developing plans while the crew was boots-on-the-ground.

\subsection{SEV as a field tool}

It should be mentioned that the SEV itself was a crucial data collection tool during EVAs. When communications were available between the SEV crew and the Science Backroom on Earth, the backroom had the ability to control the array of video and still cameras mounted on the SEV while the crew was on an EVA. This increased the efficiency of collecting data as the backroom could independently photograph the EVA site while the crew was working. Simultaneously, the backroom could watch the crew as they completed their EVA tasks, providing spatial and temporal context for the samples and data collected by each crewmember [15].

Intravehicular activities (IVAs) could be conducted by any crewmember inside the rover (either while parked at an EVA site or while the rover was moving). After an EVA was completed and the crew ingressed back into the rover, they could record IVA notes that combined video or still images with audio narration to summarize what was done on each EVA. Perhaps most useful was the technology that allowed the crew to access one of the camera interfaces (known as the GigaPan; see Lee et al. (this issue) [16]) from the SEV onboard computer and annotate the pictures directly. The GigaPan is a highresolution, $360^{\circ}$ panoramic still-frame camera that was 
mounted on the top of each SEV, and it could be controlled by either the crew or by the Science Backroom. Dozens of images are stitched together to make one GigaPan panorama, resulting in an extremely high-resolution picture of a site of interest. The crew could control this camera and could access the resulting stitched panoramas at any time from the SEV. The software interface also afforded the crew with the capability to write notes and attach them to specific places on the GigaPan image. For example, prior to egressing on an EVA, protocol dictated that the crew acquire a high-resolution GigaPan panorama of an EVA site [2]. PostEVA, the crew could bring up this image on a computer inside the SEV, and indicate exactly where they collected each sample. In this way, the Science Team received invaluable contextual information that put each sample in place with respect to each other, and with respect to the overall regional geology.

The real-time camera interface that allows the crew to annotate their EVA activities on a GigaPan image highlights the need for other capabilities that allow the crew to manage, record, and visualize their activities as they take place and their data as it is being collected. SEV navigation was managed by a Google Earth interface [7] that also contained preliminary geologic maps created by the traverse planning teams [17]. While the crews were able to access these maps from the SEV during Desert RATS 2010, being able to also edit and add to them to create a working geologic map would be very valuable. Our experiences with the image annotation functionality bring to light a need for a better, more capable mapping tool on which we can record geospatial contextual information for our observations and collected samples. We recommend the development of a Google Earth-based GIS (Geographic Information System) function in the SEV that gives the crew the capability to make a working geologic map that is editable in real-time. Post-EVA, the crew can annotate and update this map with their findings, similar to what is done with handheld electronic mapping systems in terrestrial field geology. The ability for crewmembers to collect map data while on EVA using their cuff display, and integrate it with both the GigaPan annotation system and the proposed SEV mapping system, would also greatly enhance the capacity of planetary field geologists to record geospatial contextual data and observations real-time.

\section{Lessons learned and suggestions for future tests}

The most important consideration when evaluating a tool or technology designed to assist an astronaut in planetary field geology is that these technologies should be enabling and should not cost the astronauts any "boots-on-the-ground" time. Flexibility is also crucial. A tool that can be manipulated to accomplish several different tasks is more valuable than one that is designed for a single task. For example, the rock hammer is vital for breaking off samples for return to Earth but could also be used as a scale bar in photographs or as a support for the astronaut. This eliminates the need to carry an additional tool to either show scale or to use as a walking stick.
It should also be noted that many of the concerns we raise here about the current technology, as well as our recommendations for the future, have already been integrated into the existing technology for future Desert RATS tests. They are important to document here, however, because they represent critical insights into the best practices for future planetary surface missions.

\subsection{Lessons learned: aft deck tools}

While the general concept of storing a suite of field tools on the aft deck is important and needs to preserved, we feel that improvements are needed to many of these pieces of equipment to make the tool suite optimally functional. Many of these improvements center around the development of flexible, multi-purpose technology that minimizes the amount of equipment that the crew is required to carry while on EVA. This approach, geared toward flexibility, is the one we advocate to prevent a proliferation of pieces of technology from impeding exploration.

\subsubsection{Tool construction}

The development of sturdy and multifunctional tools is critical. For example, a tool that can become a "walking stick" at any point is incredibly valuable. If the crewmember is traveling over rocky terrain and is slightly unstable in the bulky spacesuit, any tool in hand should be able to support a crewmember's weight in the event of a stumble. Video from the Apollo missions shows that the astronauts, at times, lost their balance and fell to the ground. Tools that would allow them to catch themselves and bear their weight would prevent the occurrence of this potential safety concern [3]. A rock hammer, for example, can be used for purchase and balance on a steeper slope as an astronaut climbs uphill.

Core tubes and hammers should be solid and capable of breaking off large pieces of outcrop or pushing through compacted regolith, and also easily repaired if needed. The hammers used in the Desert RATS test fit this requirement, and we recommend that style for the future. It should be noted that the mission destination would dictate the material that the tools will need to be capable of breaking. Preliminary analysis of a landing site should give astronauts and engineers an idea of what rock types to expect and their physical properties (e.g. solid bedrock or loosely consolidated materials), and therefore inform mission planners about what types of tools are needed. The tools used to work with outcrop locations could be somewhat different than those designed for a terrane mantled by regolith. In addition to dealing with science tasks related to rocks and soils of differing competency, tools should also be easy to repair and service. For example, reattaching a loose hammer head is a task that the astronaut should be able to do quickly and simply in the field.

\subsubsection{Documented Sample Bags}

The 2010 field test included only one DSB size, which was not always large enough to accommodate all samples [2]. A range of sizes (2-3) is recommended for the future. The closure method of the bags also needs improvement. 
The current system involves rolling the top of the bag downward toward the bottom of the bag and then fixing it closed with fold-down tabs. This method was faulty at times, and large samples prohibited the bag from closing properly. Large samples also had a tendency to tear or puncture the sides of the DSB, which poses a contamination risk if fragments from one sample end up escaping the bag and entering another DSB. The type of sample should also be considered when designing a sample handling system. For example, rock, soil, and core tube samples should be kept separate to keep the rocks both from being destroyed by grinding against one another and to prevent the rocks from destroying fragile soil and core tube samples, something that was not done in the 2010 Desert RATS test. Curation issues like these need to be thoroughly thought through to protect the integrity of all collected samples. The methods used in Apollo provide a robust baseline for how any extraterrestrial samples should be protected, both before and after return to Earth, and the authors recommend a similar approach for all future missions [5].

A further recommendation for future tests and missions is using radio-frequency identification (RFID) tags on every sample bag so that each sample can be electronically connected to the field notes and photographs taken at the collection site, as well as a time and location stamp. In this way, the sample is always connected to its unique identifiers and associated contextual data. We also note that there are certain types of samples that may require unique processing and tools. For example, any materials of biological interest, or that may pose a biological hazard, would require a much more complex approach to both collect and store (e.g. strict environmental controls, complete isolation from other samples, quarantine from the crew, etc.). These are issues that the Desert RATS field tests have not yet dealt with.

\subsubsection{Tool caddy}

The tool caddy represents a technology that, if perfected, could prove very useful on EVA as it both stores samples and tools while providing weight-bearing capacity. The caddy, however, has been difficult to develop, and we continue to work toward a model that satisfies these requirements while not hindering the crew in any way. Crew input from 2009 indicated that the caddy was lightweight (it utilized a soft bag to hold samples and equipment; see Fig. 3), which they liked, but they reported it was frustrating that they could not lean on the caddy while on EVA (as it was too light to provide weight-bearing capacity). The 2010 crewmembers found the most recent tool caddy model too heavy and, more critically, too tall to justify carrying it on EVA (Fig. 3). It repeatedly banged against the crew's shins and knees, as well as catching on the ground unless the crew lifted it up to at least shoulder-height (which was tiring over long distances). The weight-bearing capacity was useful, but this added weight partly contributed to the caddy being unwieldy. Though it is clearly crucial to have the capacity on EVA to carry tools and samples, the two models that have been tested in the 2009 and 2010 field tests were both less than ideal.
A successful caddy model would be one that satisfies several requirements: it should be weight-bearing; it should not catch on the ground as it is carried on EVA; and it should be easy to retrieve without forcing the astronaut to bend over once it is set down on the surface to free the user's hands for other activities. In the future, we suggest a new concept where a bag or similar container for storing samples and/or tools is clipped directly to the spacesuit. This would eliminate the need to carry anything by hand, freeing our hands for other activities, including traversing across an outcrop and manipulating cuff controls. While this would not assist the astronaut in a weightbearing capacity, the other added benefits would make up for this disadvantage. If the caddy concept moves forward, we suspect that this specific tool will be highly specified to the individual who is using it with regard to handling height and other dimensions.

\subsubsection{Sample stowage mailbox}

Intended for stowing samples after they have been collected, but before they have been loaded for return to Earth, the sample stowage mailbox (Fig. 4) was used at the conclusion of every EVA. We found the soft-sided bags hard to insert into the mailbox, as the samples within them put pressure on the edges of the soft boxes and created irregular surfaces that stuck on the sides of the mailbox. Boxes with harder sides could have mitigated this problem. The mailbox also needs to accommodate sample bags of different sizes. The largest issue associated with the stowage mailbox, however, was that it took too much EVA time to fit the samples into the mailbox. The system should be easily maneuverable with no sticking problems and be less time-consuming to use. We suggest a link with the RFID tag system that we recommend for the sample bagging system. If there is a scanner attached to the mailbox, or perhaps incorporated into the cuff control, the astronaut could scan each bag as it is stowed into the small, soft-sided boxes to ensure each sample was cataloged. In this way, there would be an electronic record of all of the mailbox contents without the need of physically organizing and grouping samples, a time-consuming process. This also negates the need to record a CFN and reviewing each sample again (which is the final task before ingress to the rover [2]), as the unique identifier information will already be there. We suggest that the crew record a CFN in the event that there are no communications with the backroom as a short summary of the EVA, but if communications were good during sample collection and the backroom recorded sample information real-time, this electronic system is efficient. One more addition that could save the crew time on EVA is a different method for weighing samples. A spring scale was used to weigh each soft-sided box before it was placed into the larger mailbox (Fig. 4). This took time, however, and was complicated by the ungainly size and shape of the soft-sided boxes. If the mailbox were equipped with a scale to measure itself automatically, it would eliminate the time the crew used to complete this task, as each increase in mass post-EVA represents the total mass of samples collected during that EVA. It should be noted that, despite these issues, the sample stowage 
mailbox is an important mission concept that ought to be developed.

\subsubsection{Recommendations for the future}

One tool that would have been extremely useful is a trenching tool capable of digging at least 6 in. beneath the surface. A common EVA task was trenching in a heavily soiled channel to look at and sample the grain size distribution in the subsurface. However, for such detailed excavation, the shovel was much too large and ungainly, especially when kneeling on the ground. The alternative was to dig with our gloved hands and the hammer handle. A trowel-like instrument is needed for this sort of work. A small tool for this purpose was included in the 2009 test, but was removed before the 2010 test because of its small size and similar function to the existing shovel tool. We suggest developing a tool that allows the modification of the existing shovel into a more flexible instrument. Including a detachable or extendable handle and modifying the shovel spade head so it can be variably sized (or including two detachable spade heads of different sizes) would be useful and would satisfy the need for a trenching tool, while also possibly acting as a "walking stick".

\subsection{Lessons learned: backpack technology}

It is recommended that many of the items discussed here should ultimately be tested with higher-fidelity suits that are needed to truly assess the effectiveness of a particular technology while on EVA. While Desert RATS does a good job of testing the technology, these tools must ultimately be tested in a higher-fidelity environment. Not only does the lack of a pressurized suit influence our view on the use of hand tools stored on the aft deck (discussed in Section 4.1), the perceived access to backpack technology is also affected. In addition, because it was easier to move our arms while wearing the shirt-sleeve backpack, it was easier to hold samples up to the cameras on either shoulder, so assessment of EVA science procedures was affected [2]. This is another issue that could be assessed in stand-alone tests using pressurized suits that would, in turn, help prepare for the primary Desert RATS field tests and, eventually, future missions.

\subsubsection{Cameras}

4.2.1.1. Camera placement. The camera placement in general was very awkward to work with. Since the cameras were mounted high on each shoulder, every picture of a sample required the crewmember to lift the sample to shoulder height and keep it there while the picture was taken. There was a five-second delay between when the user pressed the "Take Picture" button on the cuff and when the shutter actually clicked to allow the user time to properly position the sample in the approximate field of view, but once each crewmember did this action enough times, muscle memory took over and the full five seconds was not needed. In fact, the excess time proved tiring because we had to hold samples up at the awkward angle for so long and on multiple occasions on a typical EVA. In a pressurized suit, this action would be even more difficult, if not impossible. It was also physically challenging to turn our head toward the camera, even without a helmet on, and we did not have a viewfinder on the camera control system on the cuff. Because of this, positioning the sample correctly in the field of view was a matter of practice and time-consuming guesswork, with the risk of potentially missing critical science data as a result of mistakes. The approach we took to mitigating this shortcoming was, if both crewmembers were working in the same place, for one person to assist the other in composing photographs. The disadvantage to this solution was that it made what should be a single-person task into a team task, wasting time that could be used to collect more data. Adding a viewfinder to the cuff control and using the existing camera placement would better mitigate this problem, but an alternative solution we advocate would be cameras placed in different locations. We recommend a detachable camera mounted on the wrist or mounted on the chest (similar to Apollo), so the user can precisely position the camera close to the sample without the high level of physical exertion. Another possible use of a wrist camera would be to act as a hand lens. If the camera was capable of magnifying to very high-resolution, and the astronaut had real-time access to the video feed, they could have the kind of information that a hand lens gives a terrestrial field geologist.

As an alternative (or as an addition, in case the potentially vulnerable wrist camera gets broken), a helmet camera could be useful, as the user could look at what they wanted to capture, and simply push a button on the cuff control to snap the picture. The helmet camera would also solve the difficulties associated with taking context shots of the terrain surrounding the EVA location using the backpack cameras. Because the 2010 Desert RATS cameras were located above the crewmember's head and pointed slightly downward, a photo of the horizon forced the crewmember to lean uncomfortably and dangerously backward, and it was often the case that we still missed the shot because we did not have a viewfinder. Helmet cameras would ensure we are taking a picture of exactly what we are interested in.

4.2.1.2. Camera control. The fact that there were two different cameras, which were mounted on different sides of the body and at different heights, required the crew to learn to place samples in two dissimilar locations (confusing our muscle memory associated with the task). In addition to this, the crew had to photograph and describe the sample using two different cameras, a redundancy that cost both time on EVA and bandwidth as the data was sent back to the Science Team. In the future, we recommend a switch to one camera that satisfies the need for both realtime data display to the backroom and CFN documentation. This is the approach adopted for the 2011 Desert RATS field test.

An additional difficulty with the EVAIS/HD camera system was that the communications loop between the crewmember and the Science Backroom was temporarily disabled while a CFN was being taken with the HD camera, so the Science Backroom could not hear the descriptions being given about the sample. As such, the crewmember was required to provide two descriptions, and any new 
observation made during the CFN was not immediately communicated to the Science Backroom. Additionally, the EVAIS camera control interface, while simple, was in some ways awkward. The five-second delay after pressing the "Take Picture" button is an example since it cost the crew time on EVA (see [2] for the time involved in sampling protocols). Due to the fact that we had to raise our arms to shoulder height to take the picture, we lost sight of the cuff control screen, so there was no way to determine when the picture was actually taken. A sound effect (such as a loud "beep" transmitted through the headset) or similar feedback is recommended to coincide with completion of the countdown and acquisition of the image.

\subsubsection{Cuff control hardware}

As shown in Fig. 4, the size of the 2010 cuff display housing was larger than what a flight system would be. The housing was bulky and the Velcro straps did not always adequately secure it to the crewmember's arm. This was an annoyance for some crewmembers, because the display had a tendency to slide down the arm and move around during EVA operations (such as use of the rock hammer). The length of the display occupied most of a crewmembers' forearm and sometimes interfered with wrist movement and performance of EVA tasks. For 2011, the EVAIS team has reduced and optimized the size and packaging of the cuff display (Fig. 5). During an actual planetary surface mission, this cuff control would be built into one of the pressurized suit sleeves, but these lessons learned are important to apply to future analog tests and hardware development. Moreover, the rapid evolution of the cuff design is an example of the advantage of the "design-test-redesign" philosophy underlying the Desert RATS engineering efforts.

\subsubsection{Recommendations for the future}

Camera operation is being improved and simplified in 2011. The two cameras that were used in 2010 are being replaced with a single camera mounted on the backpack. This camera will operate simultaneously in multiple streams to provide both low-bandwidth, real-time video as well as HD video/still recording for CFNs. In addition, a viewfinder application on the cuff will display the camera field-of-view in real time, so that the EVA crewmember can properly point the camera and see what is being captured when recording CFN video and still images. The cuff hardware is slimmer and interferes less with crewmember EVA activities (Fig. 5). GPS positioning information has also been added to the "maps" feature on the EVAIS cuff. While on EVA, a crewmember will be able to see his/her position, as well as the position of any other EVA crewmembers and the SEV. We feel these improvements will greatly increase the effectiveness of this technology and make EVA data collection more efficient.

\section{Looking to the future}

The efforts of the 2010 Desert RATS team in developing mission flight rules, protocols, and tools yielded vast quantities of data on how existing technologies can be adapted to fit the needs of eventual manned planetary surface missions. It should be emphasized that many of the lessons learned, discussed in Section 4, have already been integrated into future Desert RATS tests, demonstrating that the input and knowledge gleaned from the experiences of the Desert RATS team have already instigated changes in future analog and real-mission activities.

From a toolkit and technology standpoint, we put forth a series of suggestions that others may consider following if they are working toward the manned exploration of other planetary bodies. Flexibility is crucial. Developing flexible equipment that can satisfy many uses will save mass on missions where every kilogram counts. Avenues should be explored that allow astronauts to clip gear and samples to their spacesuits, as this frees their hands and does not hinder them while working. An interface attached to the body (like the Desert RATS cuff control) is a very useful way of both reminding astronauts of certain procedures and controlling instrumentation such as cameras, but alternate methods of doing so should be explored (like voice activation or a head-up display). Each tool or technology taken on EVA should directly assist astronauts in their site objectives, without hindering them or slowing them down in any way.

From a science perspective, providing as much data as possible about the geologic context of a sample, from the outcrop scale to the local and regional scale of the surrounding terrain, is absolutely crucial in exploring an area. Developing the technology to make this as easy and timeefficient as possible should be a priority in the future. There are many avenues for this technology development to take place. We put forth several ideas here for this development, but recognize that this is by no means an exhaustive list:

- cameras and close-range spectral imaging instruments for spatial and compositional information;

- geochemical instruments to examine the detailed chemical or isotopic composition of a sample or outcrop;

- GPS-like positioning data for tagging an observation or sample to a specific geographic location; and

- the ability to easily and efficiently record voice and video observations, so no information is lost because the astronaut did not have an effective interface with which to do so.

If we look ahead to the potential manned exploration of asteroids, more advanced technology could aid astronaut teams in obtaining a high-resolution, real-time contextual view of the exploration target. Though it is thought that outcrops are rare on asteroids, there appears to be a wide variation among many of the asteroids that have been observed and visited. Some have diverse and blocky surfaces while others have smooth, homogenous patches $[18,19]$. Traditional geologic mapping would not be as straightforward here, but a mission designed to create a map of the surface using other methods, combined with sample collection, would look very similar to the Desert RATS mission architecture. For example, orbital or surfacedeployed Light Detection and Ranging (LiDAR) could be used to produce a topographic basemap of the asteroid. Hyperspectral and multispectral imaging cameras used by the crew would provide general compositional variability and handheld x-ray fluorescence spectroscopy could be 
conducted for a detailed investigation of areas of particular interest. These areas would then be sampled and documented using tools and protocols similar to those tested during Desert RATS.

The lessons learned from the 2010 Desert RATS mission have demonstrated the need for terrestrial analog field tests. Without the experience of multiple crewmembers with both the operational and scientific backgrounds needed to truly assess these technologies, we cannot hope to move forward in the next phase of planetary surface exploration. The amalgamation of science and engineering into integrated missions here on Earth gives teams the practice they need to work with people outside of their area of expertise, and it prepares us for the day when humans will again venture to other planetary bodies, whether it be asteroids, Mars, or our Moon.

\section{Acknowledgments}

The authors, especially those of us who served as 2010 geologist crewmembers (Young, Bleacher, Hurtado, and Rice) would like to recognize the large Desert RATS team that helped to make our experience such a positive one. In particular, the incredibly gifted and extensive Science Team working with us both prior to the test and back "on Earth" during our missions was a crucial part in our success. Specifically, Cindy Evans, Fred Horz, Gary Lofgren, John Gruener, Doug Ming, Mary Sue Bell, Kip Hodges, and above all Dean Eppler, we thank you. We also wish to thank our NASA astronaut and engineering crewmates without whom we would not have been able to complete such a successful mission: Stephanie Wilson, Stan Love, Mike Gernhardt, Chris Looper, and Aaron Hulse. Many thanks go to the many funding organizations that made this work possible. Acknowledgements go to Dr. Wendell Mendell, whose funding from the Constellation Program enabled the assembly of the world-class science team for this exercise. K. Young recognizes funding from the NASA Graduate Student Researchers Program Grant \#NNX10AK72H. J. Hurtado acknowledges funding from a 2009 NASA Field Science Analog Testing grant, NASA Minority Research and Education Program collaborative agreement \#NNX09AV09A, and NASA Lunar Science Institute cooperative agreement \#NNA09DB33A (LPI/USRA subcontract \#02173-07), which supported his participation in the 2009 and 2010 field tests. The Goddard Space Flight Center's Solar System Exploration Division provided J. Bleacher and J. Rice's support for the field test. J. Bleacher's Desert RATS supporting work and manuscript preparation funding were provided by a NASA Moon And Mars Analog Mission Activities Program grant. J. Buffington's support for the field test was provided by the NASA Desert RATS project and the Space Exploration Vehicle project.

\section{References}

[1] J. Kosmo, B. Romig, A. Ross, History and evolution of the Desert Research and Technology Studies analog, Acta Astronaut., 2012, this issue.
[2] J.M. Hurtado, K.E. Young, J. Bleacher, W.B. Garry, J. Rice, Field geologic observation and sample collection strategies for planetary surface exploration: insights from the 2010 Desert RATS geologist crewmembers, Acta Astronaut., 2012, this issue.

[3] E.M. Jones, K. Glover, Apollo Lunar Surf. J., 2010, available at $\langle$ http://history.nasa.gov/alsj/〉.

[4] E.N. Goddard, , J.H. Mackin, E.M. Shoemaker, A.C. Waters, Objectives of Apollo Geological Field Investigations and Proposal for Development of an Apollo Field Exploration System, NASA Internal Report, 1965, available at $<$ http://www.lpi.usra.edu/lunar/docu ments/ap_geology_1965.pdf $\rangle$, last accessed 6/19/21011.

[5] J.H. Allton, Catalog of Apollo Lunar Surface Geological Sampling Tools and Containers, 1989, available at $\langle$ http://cosmochemists.igpp. ucla.edu/Allton-1989-Lunar\%20Sample\%20Tool\%20Catalog.pdf $\rangle$.

[6] D.R. Williams, The Apollo Lunar Roving Vehicle, NASA, 2005, available at $\langle$ http://nssdc.gsfc.nasa.gov/planetary/lunar/apollo_lrv. html $>$.

[7] W.J. Bluethmann, Title TBD, Acta Astronaut. 2012, this issue.

[8] G. Klein, Flexecution as a paradigm for replanning, part 1, IEEE Intell. Syst. 22 (2007) 79-83.

[9] G. Klein, Flexecution, part 2: understanding and supporting flexible execution, IEEE Intell. Syst. 22 (2007) 108-112

[10] K.V. Hodges, H.H. Schmitt, A new paradigm for advanced planetary field geology developed through analog experiments on Earth, in: W.B. Garry, J.E. Bleacher (Eds.), Analogs for Planetary Exploration: Geological Society of America Special Paper, vol. 483, pp. 17-31, doi:10.1130/2011.2483(02).

[11] H.H. Schmitt, A.W. Snoke, M.A. Helper, J.M. Hurtado, K.V. Hodges, J.W. Rice Jr., Motives, methods, and essential preparation for planetary field geology on the Moon and Mars, in: W.B. Garry, J.E. Bleacher (Eds.), Analogs for Planetary Exploration: Geological Society of America Special Paper, vol. 483, pp. 1-15, doi:10.1130/ 2011.2483(01).

[12] C. Evans, M. Calaway, M. Bell, GeoLab: a habitat-based laboratory for preliminary examination of geological samples, Acta Astronaut., 2012 , this issue.

[13] J.A. Buffington, 2010 CTSD-ADV-851: 2010 Exploration Hardware Development Field Report, Tools and Equipment Branch Internal Test Report, Advanced Tools and Equipment, Planetary Surface Geology Hardware, NASA Johnson Space Center, Crew and Thermal Systems Division.

[14] D.B. Eppler, Desert RATS Science Team (41 co-authors), Desert RATS 2010 Science operations I: operational approach to managing science return from a 14 day planetary surface mission, Acta Astronaut., 2012, this issue.

[15] W.B. Garry, J.E. Bleacher, Field geology conducted from the Lunar Electric Rover, in: NASA Desert RATS 2009: Strategies for Human Surface Science Operations on the Moon, Proceedings of the 41st Lunar and Planetary Science Conference, The Woodlands, TX, Abstract 2209, 2010.

[16] P. Lee, M. Deans, D. Lees, T. Morse, Robotic planetary exploration using an interactive gigapixel panorama capturing system, Acta Astronaut., 2012, this issue.

[17] F. Horz, G.E. Lofgren, J.E. Gruener, D.B. Eppler, J.A. Skinner Jr., C.M. Fortezzo, J.S. Graf, W.J. Bluethmann, M.A. Seibert, E.R. Bell, The traverse planning process for DRATS 2010, Acta Astronaut., 2012, this issue.

[18] T. Noguchi, Hayabusa Science Team (10 co-authors), Surface morphological features of boulders on Asteroid 25143 Itokawa, Icarus 206 (2010) 319-326.

[19] P.C. Thomas, NEAR team (18 co-authors), Eros: shape, topography, and slope processes, Icarus 155 (2002) 18-37.

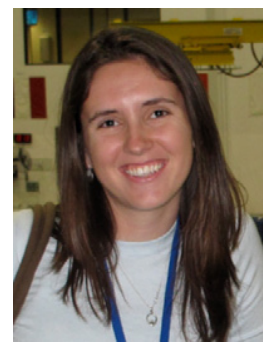

Kelsey Young is a graduate student working towards her Ph.D. in Geology in the School of Earth and Space Exploration at Arizona State University. Her research focuses on the use of terrestrial analog sites in both understanding geologic processes on other planetary bodies as well as in the context of manned space exploration. She also works on the development of handheld technology that will aid astronauts in scientific data collection in planetary field geology. Kelsey served as a Desert RATS crewmember in 2010. She earned her B.S. in Geosciences from the University of Notre Dame in 2009. 


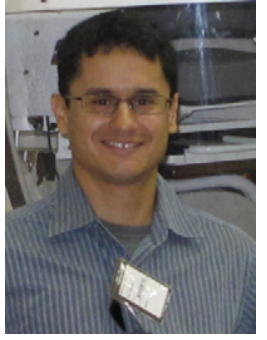

José M. Hurtado is an associate professor in the Department of Geological Sciences at the University of Texas at El Paso (UTEP) and coinvestigator with the UTEP Center for Space Exploration Technology Research. His terrestrial research focuses on the tectonic evolution of the Nepal and Bhutan Himalaya using field geology, geomorphology, thermochronology, and remote sensing. His planetary science research focuses on remote sensing studies of the lunar surface to answer questions regarding in-situ resources and the geomorphology of cratered terranes. José served on the Desert RATS science team in 2009 and as a crewmember since 2010. He earned a B.S. and M.S. in Geology from the California Institute of Technology in 1996 and a Ph.D. in Geology from the Massachusetts Institute of Technology in 2002

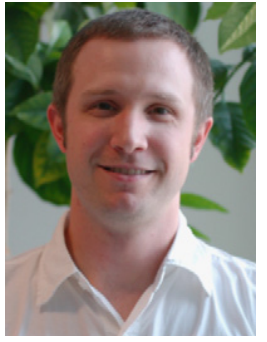

Jacob E. Bleacher is a research scientist at NASA's Goddard Space Flight Center in the Planetary Geodynamics Lab, Solar System Exploration Division. Jake's research focuses on the development and modification of planetary volcanic terrains through a combination of terrestrial field studies and spacecraft data analysis. He combines his expertise in field and planetary geology to help build and test the science capabilities of NASA's newest instrument, suit and rover technologies and has served as Desert RATS crew since 2009. He holds a B.A. in Geosciences from Franklin and Marshall College and a Ph.D. in Geological Sciences from Arizona State University.

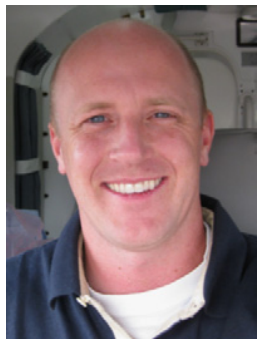

W. Brent Garry is a Research Scientist at the Planetary Science Institute. The focus of Dr. Garry's research is on the emplacement of lava flows on planetary bodies in the Solar System. He is a Participating Scientist on two NASA missions: Lunar Reconnaissance Orbiter and Dawn. Dr. Garry served as DRATS crew in 2008 and 2009 and on the science team in 2010. He completed his Ph.D. in Geology/Volcanology at the University at Buffalo, New York (2006) and holds a B.S in Geology from the College of William and Mary, Virginia (1999) and an M.S. in Geology from the University of Kentucky (2001).

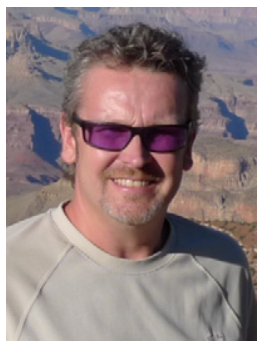

Scott Bleisath is a Lead Systems Engineer a NASA Glenn Research Center (GRC). He started working for NASA as a co-operative education student 1986-1987 at NASA Johnson Space Center (JSC). After graduating from college, Scott worked at NASA JSC for 20 years as a lead Extravehicular Activity (EVA) Officer in Mission Control, where he led spacewalk operations for multiple Space Shuttle and International Space Station missions. Scott currently works at NASA GRC and is developing electronics and computer displays for NASA's next generation of astronaut space suits. On Desert RATS, he is leading a team that is testing an EVA Information System and wrist display to provide maps and procedures to astronauts and a high definition space suit video camera to record geology field notes during the DRATS space walks. Scott has a B.S. in Aerospace Engineering from the University of Cincinnati and an M.S. in Industrial Engineering from the University of Houston.

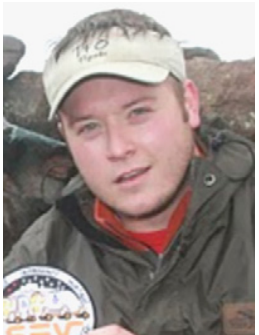

Jesse Buffington is a Johnson Space Center Civil Servant Project Manager in the Tools and Equipment Branch (EC7) of NASA JSC's Engineering Directorate, Crew and Therma Systems Division. Jesse started at JSC certifying EVA Flight Hardware for the Space Shuttle Program as part of the Orbiter Thermal Protection System Repair (TPS Repair) efforts created after the Columbia accident. Concurrently with this effort, he began the "Advanced Tools" Project in EC7, seeking to identify and develop capabilities necessary to enable mission objectives for destinations beyond Low Earth Orbit. Continuing his emphasis on exploration related hardware development, Jesse is also preparing for 2011 field tests with the Desert RATS analog and the NASA Extreme Environments Mission Operations (NEEMO) submerged facility. He graduated with a B.S. from the University of Arkansas in 2007. Away from work, Jesse spends time with his wife Shelly and their two Shetland Sheepdogs as well as managing and operating a privately owned machine shop that he founded in 2008 .

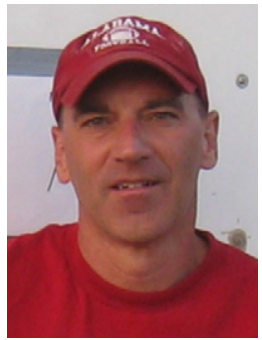

Dr. Jim W. Rice is an Astrogeologist working at NASA Goddard Space Flight Center. A 2010 Desert RATS crewmember, Jim is also a CoInvestigator on both Mars Rover Missions (Spirit and Opportunity) and has worked on the Mars Odyssey and Mars Polar Lander Missions. He is also an Associate Project Scientist on the Lunar Reconnaissance Orbiter Project. His research specializes on the surface geology and history of water on Mars. Jim also conducts planetary analog field geology investigations in the Antarctic Arctic, Iceland, Hawaii, Arizona, and California. He has been highly involved in Mars landing site selection and certification for all Mars Missions since Mars Pathfinder in 1995. Dr. Rice received his B.S. in Geology from the University of Alabama, his M.S. in Geosciences from Northeast Louisiana University, and his Ph.D. from Arizona State University. 